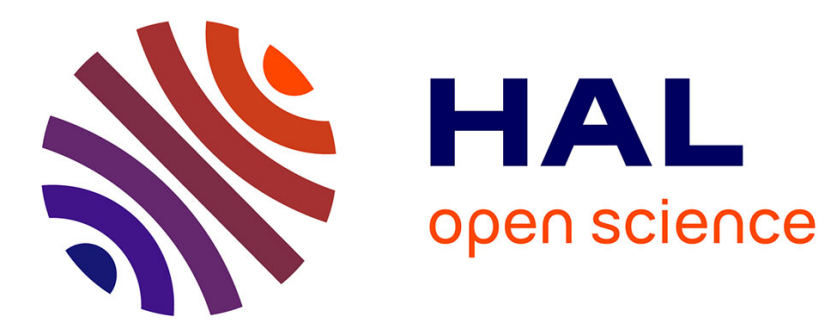

\title{
Identification of Lamination Stack Properties: Application to High Speed Induction Motors
}

Guillaume Mogenier, Régis Dufour, Guy Ferraris Besso, Lionel Durantay, Nicolas Barras

\section{- To cite this version:}

Guillaume Mogenier, Régis Dufour, Guy Ferraris Besso, Lionel Durantay, Nicolas Barras. Identification of Lamination Stack Properties: Application to High Speed Induction Motors. IEEE Transactions on Industrial Electronics, 2010, 57 (1), pp.281-287. 10.1109/TIE.2009.2029522 hal-00485145

\section{HAL Id: hal-00485145 \\ https://hal.science/hal-00485145}

Submitted on 13 Feb 2019

HAL is a multi-disciplinary open access archive for the deposit and dissemination of scientific research documents, whether they are published or not. The documents may come from teaching and research institutions in France or abroad, or from public or private research centers.
L'archive ouverte pluridisciplinaire HAL, est destinée au dépôt et à la diffusion de documents scientifiques de niveau recherche, publiés ou non, émanant des établissements d'enseignement et de recherche français ou étrangers, des laboratoires publics ou privés. 


\title{
Identification of Lamination Stack Properties: Application to High-Speed Induction Motors
}

\author{
Guillaume Mogenier, Régis Dufour, Guy Ferraris-Besso, Lionel Durantay, and Nicolas Barras
}

\begin{abstract}
In order to predict the lateral rotordynamics of a high-speed induction motor, an optimization procedure is used for identifying the dynamic behavior of the magnetic core made of a lamination stack, tie rods, and short-circuit rods. Modal parameters predicted by a finite-element model based on beam elements and measured on induction motors are included in modal error functions contained in a functional. The minimization of this functional by using the Levenberg-Marquardt algorithm permits extracting the equivalent constitutive properties of the lamination stack for several rotors of different sizes. Finally, the size effect on the constitutive properties identified is discussed.
\end{abstract}

Index Terms-Finite-element (FE) methods, parameter identification, squirrel-cage motors, vibrations.

\section{INTRODUCTION}

$\mathbf{T}$ HIS PAPER focuses on the squirrel-cage induction motor known as the Moteur Grande Vitesse (MGV) in the 3 to $30 \mathrm{MW}$ range from 6000 to $18000 \mathrm{r} / \mathrm{min}$, used for critical applications, particularly motocompressors, in the oil and gas industry. This paper continues from an International Conference on Electronic Materials 2008 conference paper dealing with other experiments performed on several MGVs to evaluate a possible relation between identified parameters and rotor sizes, i.e., magnetic core length $L_{\text {fer }}$ (Fig. 1) and diameter $D_{\text {fer }}$ (Fig. 2). As shown in Fig. 1, an MGV is mainly composed of two steel shaft ends and full-depth laminations held together by steel tie rods. The squirrel cage consists of copper shortcircuit rods distributed at the periphery of the core (Fig. 2) and linked to two bronze alloy rings placed at both ends of the stack. The stack and the rings are tightened by the tie rods, also distributed at the periphery of the core and screwed into the shaft ends. This configuration is proposed as full-depth laminations considerably reduce the eddy-current circulation along the longitudinal axis in the core of the magnetic part and thus eliminate flux saturation. Consequently, the MGV is more

G. Mogenier, R. Dufour, and G. Ferraris-Besso are with the Centre National de la Recherche Scientifique, Institut National des Sciences Appliquées de Lyon, Laboratoire de Mécanique des Contacts et des Structures, Unite Mixte de Recherche 5259, Université de Lyon, 69621 Villeurbanne, France (e-mail: guillaume.mogenier@insa-lyon.fr; regis.dufour@insa-lyon.fr; guy.ferraris@insa-lyon.fr).

L. Durantay and N. Barras are with the Department of Research and Development, Converteam SAS, Rotating Machines Division, 54250 Champigneulles, France (e-mail: lionel.durantay@converteam.com; nicolas. barras@converteam.com).

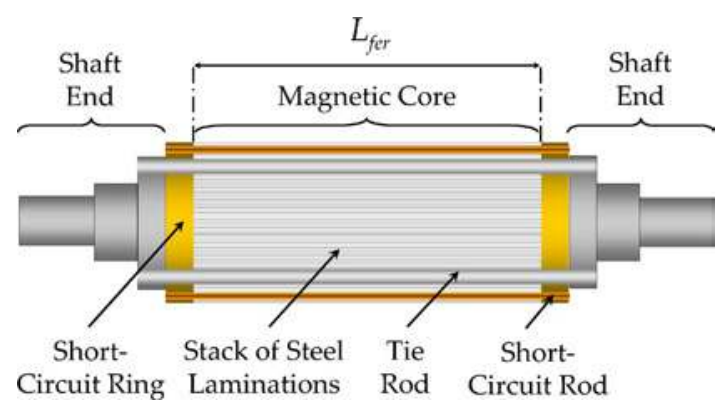

Fig. 1. Diagram of a squirrel-cage induction motor.

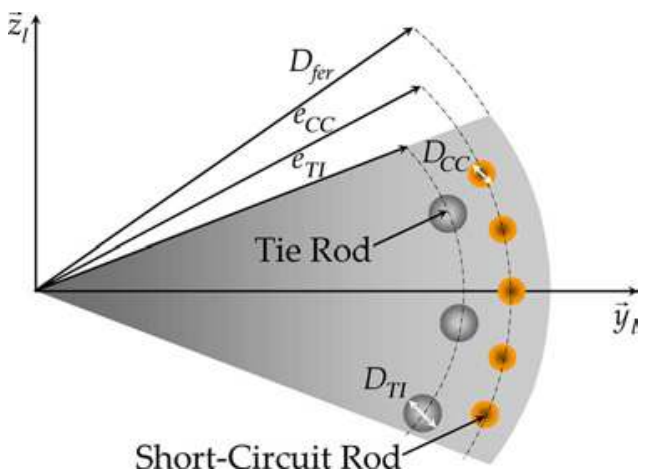

Fig. 2. Cross section of the magnetic core of a squirrel-cage induction motor.

compact and efficient than widely used solid rotors with shrink fitted laminations. It also prevents problems such as electrical loss, heat dissipation, and loss of adhesion between the components although it is difficult to predict its dynamic behavior. The problem of modeling the magnetic core and the stacks of fulldepth laminations has been given little attention in scientific papers. References [1] and [2] suggested homogenized bending rigidity for the entire magnetic core cross section by adding the bending rigidity of each cross section component. McClurg [3] deals with a stiff shaft design for a squirrel-cage rotor, but the bending rigidity of the magnetic core is not described. However, many authors have attempted to predict the dynamic behavior of induction machine rotors with a laminated core mounted on a solid shaft, i.e., laminations around central hole. The stiffening effects of the laminated core are not easy to assess and often require identification via modal testing. Ede et al. [4] assess the influence of leading design parameters, such as stack length, on the natural frequencies of a high-speed permanent magnet brushless machine by using a 3-D finiteelement (FE) model. Garvey et al. [5] present the advantages of a branched model for a laminated rotor. The laminations 
are considered as several annular ring subsets linked together by elastic connections. Moreover, the subsets are elastically connected to the shaft. Chen et al. [6] consider an equivalent Young modulus for the stack and use a branched beam model for the magnetic core. The authors of many papers have dealt with a coefficient known as stacking factor that modifies the value of the mass of the lamination stack. In addition, its weak Young modulus is due to resin or varnish layers on the interfaces between consecutive laminations. Garvey et al. [5] consider the lamination stack as an orthotropic material whose elastic strain-stress relation takes into account the lamination material and the mean flexibilities (shear and compressive) of the lamination interfaces. Kim and Kim [7] show that the lamination pressure has considerable influence on the lateral natural frequencies of a rotor. An equivalent diameter and lumped masses are considered for modeling the entire magnetic core.

In identification procedures, an optimization algorithm is used so that the optimization parameters of an FE model make predicted data tend toward target values, i.e., measured data. Such algorithms can use modal data for updating and then identifying the FE model parameters. Modal error functions are defined to quantify the difference between predicted and measured natural frequencies and mode shapes. Lee and Kam [8] use a common modal error function based on natural frequencies in order to identify the properties of laminated composite plates. Cugnoni [9] proposes two original modal error functions based on the diagonal and extra-diagonal terms of the modal assurance criterion matrix. Feng et al. [10] use a more classical modal error function, based on the difference between the components of predicted and measured mode shapes, to identify the material parameters of concrete dams. These functions are combined in a functional whose minimization requires its derivatives, with respect to the optimization parameters, that depend on eigenelement derivatives. Eigenvalue derivatives are obtained from the relation given by [11] whereas eigenvector derivatives can be computed by using approximate or exact methods [12]. Min et al. [13] compare different algorithms designed to achieve this.

In this paper, an FE model of an induction rotor is presented with particular attention given to modeling the magnetic core. It is based on the structural dynamics theory described in [14] and therefore uses beam FE in order to limit the degrees of freedom (DOF). Under certain assumptions, tie and shortcircuit rods can also be modeled with beam FE. Even if a beam-based model is used, the constitutive properties of the stack are defined such that the shear and the Young moduli are independent in order to take into account its orthotropic properties [5]. The lateral dynamic behavior of the stack is then identified by performing experimental modal analyses on several rotors of different sizes. The identification strategy presented in [15] consists in minimizing the difference between the measured and the predicted modal data provided by the FE model, by using the Levenberg-Marquardt algorithm [16], [17]. Identification results are discussed to evaluate a possible relation between the equivalent constitutive properties of the lamination stack and the magnetic core length and diameter.

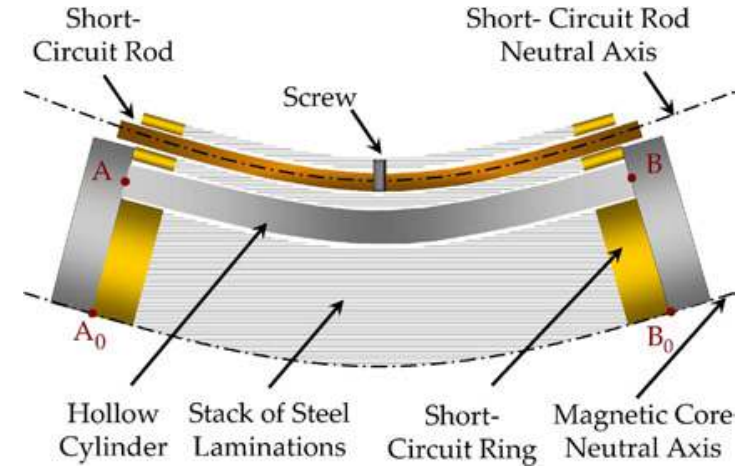

Fig. 3. Short-circuit rod in bending-kinematic assumption.

\section{FE MOdEL}

The predicted modal data emanate from the eigenvalue equation of the undamped FE model based on the structural dynamic theory, described in [14], for each mode $k=1, \ldots, m$

$$
\left(K-\widetilde{\omega}_{k}^{2} M\right) \widetilde{\varphi}_{k}=0, \quad \text { with } K=K_{S}+K_{P}
$$

where $\widetilde{\omega}_{k}, \widetilde{\varphi}_{k}$ are the $k$ th angular frequency and associated mode shape, respectively. $K_{S}$ is the structural stiffness matrix, $K_{P}$ is the stress stiffening matrix if a possible axial prestressing force acts on the beam FE [18]. $M$ is the mass matrix due to the translation and rotatory inertia, the latter being classically neglected for slender structures. Shear strain and rotatory inertia are taken into account in the Timoshenko in-plane beam FE containing 2 DOF per node: one translation and one rotation. Thus, a shear correction factor dependent on the shape of the cross section is used as in [19].

Free-free boundary conditions are taken into account by adding low-stiffness springs $(10 \mathrm{~N} / \mathrm{m})$ at each boundary node. Disks and landing rings are considered as lumped masses. To identify the stiffness parameters of the lamination stack, specific assumptions must be considered to model the tie and the short-circuit rods. The latter can be modeled as beams of diameter $D_{\mathrm{CC}}$ (Fig. 2) whose neutral axes coincide with the neutral axis of the magnetic core. These rods are fastened at middle of the magnetic core by screws and their ends can slide in the short-circuit rings, as shown in Fig. 3. Thus, each FE of the discretized magnetic core is connected to two consecutive nodes its elementary matrices being the sum of those of the short-circuit rods and the lamination stack. Furthermore, the tie rods are modeled as a one FE equivalent hollow cylinder clamped at nodes $A_{0}$ and $B_{0}$ (see Fig. 2). Let $D_{\text {Out }}^{\mathrm{TI}}$ and $D_{\text {In }}^{\text {TI }}$ be its outer and inner diameters, respectively, and simply calculated by considering surface $S_{\mathrm{TI}}$ and second moment of area $I_{\mathrm{TI}}$ of the tie rods

$$
D_{\text {Out }}^{\mathrm{TI}}=\left(\frac{8 I_{\mathrm{TI}}}{S_{\mathrm{TI}}}+\frac{2 S_{\mathrm{TI}}}{\pi}\right)^{\frac{1}{2}}, \quad D_{\mathrm{In}}^{\mathrm{TI}}=\left(\frac{8 I_{\mathrm{TI}}}{S_{\mathrm{TI}}}-\frac{2 S_{\mathrm{TI}}}{\pi}\right)^{\frac{1}{2}} .
$$

The screwing torque ensures the stress stiffening in the tie rods and the prestressing in the lamination stack. The stress stiffening effect is taken into account by the $K_{P}$ matrix (1) only for the hollow cylinder. The measurement is performed 
on the assembled structure; therefore, the identified parameters implicitly take into account the prestressing which is of the same magnitude for each rotor presented in Section III. Since the short-circuit rings, the lamination stack and the shaft ends connected to each ring are drilled at their periphery for the short-circuit rods and tie rods, (2) is also used to model the cross section of these elements.

\section{Optimization StRATEGy}

The equivalent constitutive properties of the lamination stack are denoted $x \in \mathbb{R}^{n}$ so that $\{x\}_{p=1, \ldots, n}$. The optimization parameters, stemming from the following doublet:

$$
\{E, G\}
$$

where $E$ and $G$ are the Young and the shear moduli, respectively, and they are also independent for modeling the anisotropy of the lamination stack, as described in [5]. The optimization parameter number is therefore equal to two, i.e., $(n=2)$. The identification strategy consists in minimizing the difference between the predicted and measured modal data.

\section{A. Modal Error Functions}

An optimization algorithm is used so that the optimization parameters of an FE model make the predicted data tend toward target values, i.e., measured data. Let $x^{i}$ be the vector of optimization parameters $x$ at iteration $i$. Let us assume that all the variables of the FE model depend on $x^{i}$. Let $\widetilde{\omega}_{k}^{i}$ and $\omega_{k}$ be the predicted and measured natural frequencies, respectively. Let $\widetilde{\varphi}_{k}^{i}$ and $\varphi_{k}$ be their associated mode shapes projected on the same spatial basis. The first modal error function is based on the difference between measured natural frequencies $\omega_{k}$ and predicted natural frequencies $\widetilde{\omega}_{k}^{i}$ for each mode shape $k(k=$ $1,2, \ldots, m)[8]$

$$
F_{k}^{\omega}\left(x^{i}\right)=\frac{\widetilde{\omega}_{k}^{i}}{\omega_{k}}-1
$$

When performing modal tests, the MAC method is that most commonly used for estimating the correlation of measured mode shapes. It provides a matrix $M_{j l}=\operatorname{MAC}\left(\widetilde{\varphi}_{j}, \varphi_{l}\right)$ of scalar products between two sets of mode shapes $\varphi_{l}(l=$ $1,2, \ldots, m)$ and $\widetilde{\varphi}_{j}(j=1,2, \ldots, m)$, denoted as follows:

$$
\operatorname{MAC}\left(\widetilde{\varphi}_{j}, \varphi_{l}\right)=\frac{\left(\widetilde{\varphi}_{j} \cdot \varphi_{l}\right)^{2}}{\left(\widetilde{\varphi}_{j} \cdot \widetilde{\varphi}_{j}\right)\left(\varphi_{l} \cdot \varphi_{l}\right)}
$$

Usually, two mode shapes $\varphi_{l}$ and $\widetilde{\varphi}_{j}$ are considered identical if $M_{j l}$ is higher than 0.9 , close together if $M_{j l}>0.7$ or 0.8 depending on measurement accuracy. For each pair of measured and predicted modes $k=1,2, \ldots, m$, let a second modal error function be defined as follows:

$$
F_{k}^{\varphi_{D}}\left(x^{i}\right)=1-\operatorname{MAC}\left(\widetilde{\varphi}_{k}^{i}, \varphi_{k}\right)
$$

On the strength of the orthogonality property of the mode shapes, a perfect correlation between two sets of mode shapes requires that all the extra-diagonal terms of the MAC matrix vanish. Thus, a third modal error function can be defined [9]

$$
F_{k}^{\varphi_{\mathrm{ED}}}\left(x^{i}\right)=\sum_{j=1, j \neq k}^{m}\left|\operatorname{MAC}\left(\widetilde{\varphi}_{j}^{i}, \varphi_{k}\right)-\operatorname{MAC}\left(\varphi_{j}, \varphi_{k}\right)\right| .
$$

A fourth modal error function can be defined by estimating the difference between two sets of mode shapes by simply calculating the sum of the absolute values of differences between the modal vector components. However, it is essential that the pair of measured and predicted mode shapes are normalized equally and signed so that the larger component is equal to unity

$$
F_{k}^{\varphi \mathrm{EC}}\left(x^{i}\right)=\frac{1}{r} \sum_{j=1}^{r}\left|\frac{\left(\widetilde{\varphi}_{k}^{i}\right)_{j}}{\max _{l}\left(\left(\widetilde{\varphi}_{k}^{i}\right)_{l}\right)}-\frac{\left(\varphi_{k}\right)_{j}}{\max _{l}\left(\left(\varphi_{k}\right)_{l}\right)}\right|
$$

where $j$ stands for the $j$ th vector component whereas $r$ is the number of vector components. The identification method is then reduced to the minimization of a global error functional $f\left(x^{i}\right)$ with respect to optimization parameters vector $x^{i}$ including $n$ parameters. The global error functional can be expressed as a function of the previous four modal error functions

$$
f\left(x^{i}\right)=\frac{1}{2}\left\|F^{\operatorname{Tot}}\left(x^{i}\right)\right\|^{2}=\frac{1}{2} \sum_{k=1}^{q}\left[F_{k}^{\text {Tot }}\left(x^{i}\right)\right]^{2}, \quad q=4 \times m
$$

with

$$
F^{\mathrm{Tot}}\left(x^{i}\right)=\left[\alpha^{\omega} F^{\omega}, \alpha^{\varphi_{D}} F^{\varphi_{D}}, \alpha^{\varphi_{\mathrm{HD}}} F^{\varphi_{\mathrm{HD}}}, \alpha^{\varphi_{\mathrm{Ec}}} F^{\varphi_{\mathrm{Ec}}}\right]^{\mathrm{T}} .
$$

Weight coefficients $\alpha$ (10) permit controlling the order of each function as they are very different from each other. Thus, a weighting coefficient linked to a modal error function is defined as the inverse of the mean value of this modal error function components calculated at the first iteration, i.e., $i=0$. We propose defining each weight coefficient $\alpha$ (10) as follows:

$$
\alpha=1 / \overline{F\left(x^{0}\right)} \text {. }
$$

To avoid numerical conditioning problems (a $10^{12}$ ratio between the Young modulus and the Poisson ratio), particularly for the Jacobian matrix $J\left(x^{i}\right)(12)$, the $x^{i}$ are defined relative to reference values such as the initial values of optimization parameters $x^{0}$. This functional is minimized by using the Levenberg-Marquardt algorithm, which is generally acknowledged as being very robust and highly efficient in a wide range of problems

$$
\mid \begin{array}{l|l}
x^{0}, \lambda_{0} \text { given } & \nabla f=J\left(x^{i}\right)^{\mathrm{T}} F^{\mathrm{Tot}} \\
d_{i}=-\left(H_{i}+\lambda_{i} I\right)^{-1} \nabla f, & H_{i} \approx J\left(x^{i}\right)^{\mathrm{T}} J\left(x^{i}\right) \\
x^{i+1}=x^{i}+\rho_{i} d_{i}
\end{array}
$$

where $I$ and $H_{i}$ are, respectively, the identity and the approximate Hessian matrices of size $[n \times n]$. If $\lambda_{i} \rightarrow+\infty$, the method tends toward the steepest descent method, whereas if $\lambda_{i} \rightarrow 0$, the method tends toward to the Gauss-Newton method. Updating damping parameter $\lambda_{i}$ is done by calculating 
a "gain factor," i.e., the ratio of the $f\left(x^{i}\right)$ decrease over the $F^{\text {Tot }}$ decrease, as vector $F^{\text {Tot }}$ is expanded by a Taylor series. Descent step $\rho_{i}$ can be obtained by a "Line Search" algorithm. The method proposed by [16] is used here by setting $\rho_{i}=1$ and frequently updating $\lambda_{i}$. This results in smoother performance and faster convergence than that achieved by Marquardt's updating strategy.

\section{B. Eigenderivatives}

The Jacobian matrix $J\left(x^{i}\right)$ (of size $[q \times n]$ ) of the total error vector $F^{\text {Tot }}$ implicitly depends on eigenelements $\lambda_{k}$ and $\widetilde{\varphi}_{k}$ defined in (1), $\lambda_{k}$ being equal to the square of the $k$ th angular frequency $\omega_{k}$. Assuming that the eigenvectors are normalized with respect to the mass matrix $M$

$$
\widetilde{\varphi}_{k}^{\mathrm{T}} M \widetilde{\varphi}_{k}=1, \quad k=1, \ldots, m .
$$

Taking partial derivatives of (1) and (13) with respect to an optimization parameter $x_{p}$ yields the following governing equations for eigenvector derivatives [11]:

$$
\begin{aligned}
A_{k} \frac{\partial \widetilde{\varphi}_{k}}{\partial x_{p}} & =P_{k} \\
\widetilde{\varphi}_{k}^{\mathrm{T}} M \frac{\partial \widetilde{\varphi}_{k}}{\partial x_{p}} & =Q_{k} \quad \text { with } \quad Q_{k}=-\frac{1}{2} \widetilde{\varphi}_{k}^{\mathrm{T}} \frac{\partial M}{\partial x_{p}} \widetilde{\varphi}_{k}
\end{aligned}
$$

where

$$
A_{k}=\left(K-\lambda_{k} M\right), \quad P_{k}=-\left(\frac{\partial K}{\partial x_{p}}-\frac{\partial \lambda_{k}}{\partial x_{p}} M-\lambda_{k} \frac{\partial M}{\partial x_{p}}\right) \widetilde{\varphi}_{k} .
$$

By premultiplying (14) by $\widetilde{\varphi}_{k}^{\mathrm{T}}$ and substituting (1) and (13), the eigenvalue derivative with respect to $x_{p}$ is obtained from the relationship

$$
\frac{\partial \lambda_{k}}{\partial x_{p}}=\widetilde{\varphi}_{k}^{T}\left(\frac{\partial K}{\partial x_{p}}-\lambda_{k} \frac{\partial M}{\partial x_{p}}\right) \widetilde{\varphi}_{k} .
$$

The partial derivatives of global mass and stiffness matrices with respect to $x_{p}$ are easily obtained by differentiating elementary mass and stiffness matrices (1). Concerning the eigenvector derivatives, the problem is that (14) is not invertible since the $A_{k}$ matrix is of rank $(m-1)$. The complete modal method assumes that the $k$ th eigenvector derivative with respect to $x_{p}$ can be expressed as follows:

$$
\frac{\partial \widetilde{\varphi}_{k}}{\partial x_{p}}=\sum_{j=1}^{m} c_{j} \widetilde{\varphi}_{j}
$$

Substituting (18) and (14) and premultiplying by $\widetilde{\varphi}_{k}^{\mathrm{T}}$ gives

$$
c_{j}=\frac{\widetilde{\varphi}_{j}^{\mathrm{T}} \cdot P_{k}}{\lambda_{j}-\lambda_{k}}, \quad j \neq k .
$$

Equation (19) shows that the eigenvector derivative has a unique expression (Linear combination) in terms of all the system's eigenvectors, excluding the $k$ th one

$$
\frac{\partial \widetilde{\varphi}_{k}}{\partial x_{p}}=\sum_{\substack{j=1 \\ j \neq k}}^{m} c_{j} \widetilde{\varphi}_{j}+c_{k} \widetilde{\varphi}_{k} \equiv V_{k}+c_{k} \widetilde{\varphi}_{k} .
$$

TABLE I

Size ANd INERTIAL CharaCteristics OF THE TESTED Rotors

\begin{tabular}{cccccc}
\hline \hline Rotor & $\# 1$ & $\# 2$ & $\# 3$ & $\# 4$ & $\# 5$ \\
\hline Total Weight $(\mathrm{kg})$ & 240 & 830 & 1969 & 998 & 1704 \\
Total Length $(\mathrm{m})$ & 1.15 & 2.02 & 2.61 & 2.03 & 2.24 \\
$D_{\text {fer }}(\mathrm{m})$ & 0.248 & 0.346 & 0.439 & 0.346 & 0.439 \\
$L_{\text {fer }}(\mathrm{m})$ & 0.375 & 0.610 & 0.910 & 0.850 & 0.750 \\
\hline \hline
\end{tabular}

By substituting (20) and (15), $c_{k}$ can be obtained by

$$
c_{k}=Q_{k}-\widetilde{\varphi}_{k} M V_{k} .
$$

This method is costly in terms of computation time because it requires knowing all the eigenvectors of the system. Thus, by substituting (20) in (14), [12] proposes removing the singularity of $A_{k}$ by cancelling a component of $V_{k},(14)$ becomes invertible and part of the solution $V_{k}$ is obtained. The complete solution is then given by (20) and (21). This method requires as many inversions of matrix $A_{k}$ of size $[m \times m]$ as eigenvector derivatives needed. However, MGV rotors are considered as medium systems and the inversion of such matrices is not costly in terms of CPU time.

\section{INDUSTRIAL APPLICATIONS}

Experimental modal analyses were conducted on five MGV rotors denoted as Rotor\#1 through Rotor\#5 whose total lengths and weights are presented in Table I. Rotor\#1 is the rotor presented in [15]. Length $L_{\text {fer }}$ and diameter $D_{\text {fer }}$ (Figs. 1 and 2 ) of the core vary from rotor to rotor, as shown in Table I. The rotors were hung from a crane via a flexible sling and a swivel hoist ring to achieve free-free boundary conditions at best. The hanging rotors were radially excited along a meridian line with an impulse force hammer (KISTLER Type 9726A20000 of mass $0.5 \mathrm{~kg}$ ), with the transmitted force being measured by a load cell. A steel impact tip was used to observe a large frequency spectrum. Meridian lines were discretized in fine experimental meshes to establish accurate mode shapes. Sets of $62,81,73,68$, and 89 measurement points were defined for Rotor\#1 through \#5, respectively. The successive accelerances obtained with a dynamic analyzer permit evaluating the measured natural frequencies. The mode shapes are plotted by exploring the imaginary part of the accelerances.

Identification procedures were performed by updating the FE models of each rotor, as described in Sections II and III. A minimum of four modes were retained to ensure sufficient measured modal data to preserve the dynamics of the rotors studied. Poisson ratio was fixed at 0.28 , as in [6]. Thus, for each rotor, the optimization problem contains two unknowns $\{E, G\}$, the Young and shear moduli, respectively. Five sets of constitutive properties have been identified and are presented in Table II. The normalized values are reported and defined as follows:

$$
E^{*}=\frac{E}{\bar{E}}, \quad G^{*}=\frac{G}{\bar{G}}, \quad L^{*}=\frac{L_{\mathrm{fer}}}{\bar{L}_{\mathrm{fer}}}, \quad D^{*}=\frac{D_{\mathrm{fer}}}{\bar{D}_{\mathrm{fer}}}
$$

where $\bar{E}$ and $\bar{G}$ are the mean values of the identified Young and shear moduli, respectively. Consequently, $\bar{L}_{\text {fer }}$ and $\bar{D}_{\text {fer }}$ are 
TABLE II

IDENTIFIEd Dimensionless EQuivalent Constitutive Properties

\begin{tabular}{cccccc}
\hline \hline Rotor & $\# 1$ & $\# 2$ & $\# 3$ & $\# 4$ & $\# 5$ \\
\hline$E^{*}$ & 0.65 & 1.05 & 0.83 & 1.59 & 0.88 \\
$G^{*}$ & 0.43 & 0.27 & 0.74 & 2.21 & 1.34 \\
\hline \hline
\end{tabular}

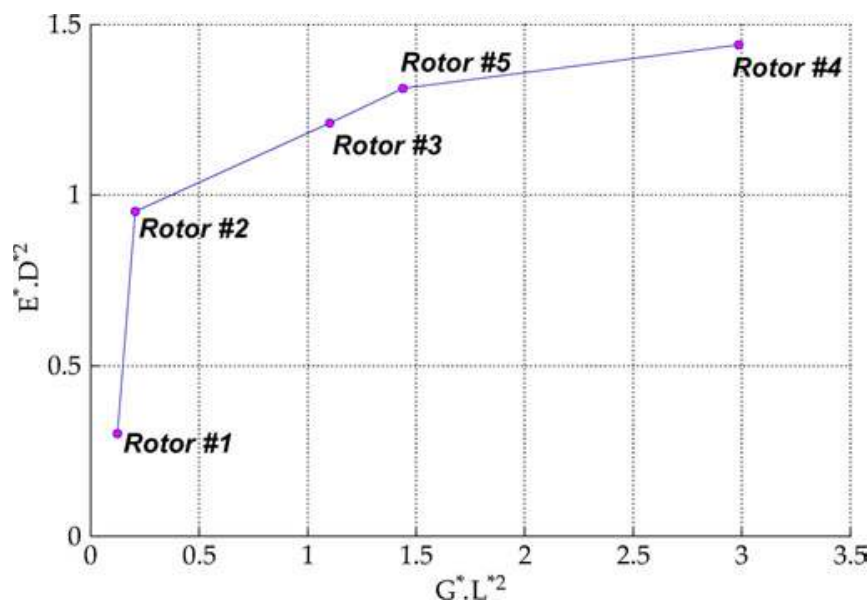

Fig. 4. Evolution of the $\beta$ parameter versus the $\sigma$ the parameter.

the mean values of the magnetic core lengths and diameters in order to obtain dimensionless variables.

To evaluate a correlation between the identified constitutive properties and the rotor sizes, the first step is achieved by using coefficient $a$ (23) which characterized the shear effect in the FE model, as defined in [18]. It can be seen as the ratio of the bending and the shearing rigidities and tends to a low value for slender structures and to a high value for monolithic structures. Let $\beta$ and $\sigma$ be the numerator and the denominator, respectively, of coefficient $a$. They are defined as follows:

$$
a=\frac{12 E I}{G S_{r} L^{2}} \sim \frac{E^{*} D^{* 2}}{G^{*} L^{* 2}}=\frac{\beta}{\sigma}
$$

where $L, S_{r}, I$ are the characteristic length, reduced area, and second moment of inertia of the magnetic core, respectively. Fig. 4 shows the evolution of parameter $\beta$ versus parameter $\sigma$. This representation shows that the identified bending rigidity of the magnetic core increases with the associated identified shear rigidity. Moreover, the identified bending rigidity seems to be very sensitive to low values of the identified shear rigidity while it tends to increase slowly for high values of the identified shear rigidity.

The second step is carried out by plotting the normalized identified Young modulus $E^{*}$ weighted by $L^{* 2}$ as a function of $D^{* 2} L^{*}$, a classical parameter used in electromagnetic design. Fig. 5 shows that $E^{*} / L^{* 2}$ decreases quasi-linearly with the $D^{* 2} L^{*}$ parameter. This behavior probably signifies an exponential decrease, since the normalized identified Young modulus $E^{*}$ cannot be negative for a high value of $D^{* 2} L^{*}$. This means that for a given parameter $D^{* 2} L^{*}$, a Young modulus can be found for the lamination stack. Therefore, a shear modulus can be associated, by using the curve shown in Fig. 4.

The first four predicted and measured natural frequencies are given in Table III while the predicted and measured lateral

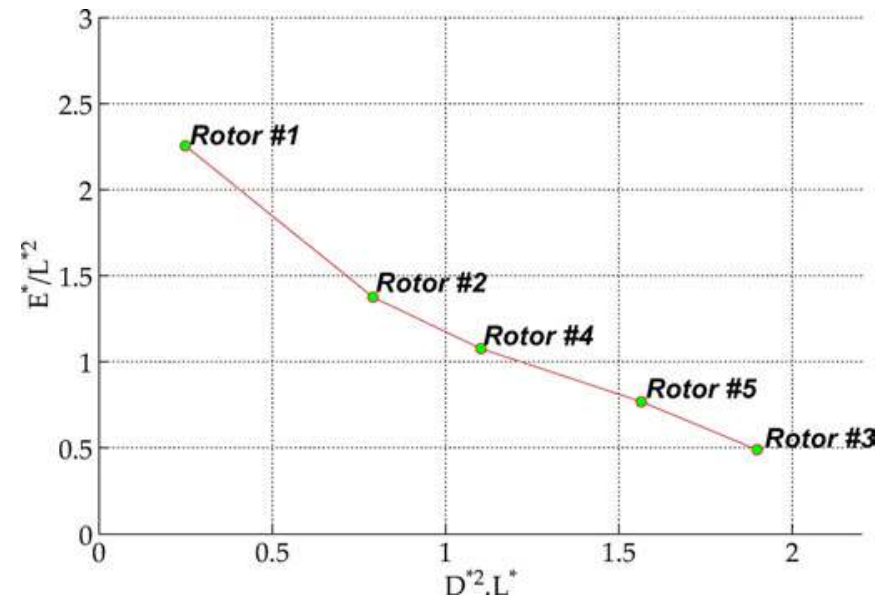

Fig. 5. Evolution of the normalized identified Young modulus weighted by $L^{* 2}$ versus the $D^{* 2} L^{*}$ parameter.

TABLE III

First Four Predicted ANd MEASUREd NATURAL FreQUENCIES

\begin{tabular}{|c|c|c|c|c|}
\hline Rotor\#1 & $1^{s t}$ & $2^{n d}$ & $3^{r d}$ & $4^{t h}$ \\
\hline Measured & $856 \mathrm{~Hz}$ & $1230 \mathrm{~Hz}$ & $1713 \mathrm{~Hz}$ & $2330 H z$ \\
\hline Predicted & $844 \mathrm{~Hz}$ & $1285 \mathrm{~Hz}$ & $1741 \mathrm{~Hz}$ & $2279 \mathrm{~Hz}$ \\
\hline Error & $1.4 \%$ & $4.5 \%$ & $1.6 \%$ & $2.2 \%$ \\
\hline Rotor\#2 & $1^{\text {st }}$ & $2^{n d}$ & $3^{r d}$ & $4^{t h}$ \\
\hline Measured & $426 H z$ & $610 \mathrm{~Hz}$ & $953 H z$ & $1243 H z$ \\
\hline Predicted & $423 \mathrm{~Hz}$ & $677 \mathrm{~Hz}$ & $898 \mathrm{~Hz}$ & $1216 \mathrm{~Hz}$ \\
\hline Error & $0.9 \%$ & $10.9 \%$ & $5.7 \%$ & $2.2 \%$ \\
\hline Rotor\#3 & $I^{s t}$ & $2^{n d}$ & $3^{r d}$ & $4^{t h}$ \\
\hline Measured & $247 \mathrm{~Hz}$ & $440 \mathrm{~Hz}$ & $766 \mathrm{~Hz}$ & $1143 \mathrm{~Hz}$ \\
\hline Predicted & $242 H z$ & $470 \mathrm{~Hz}$ & $749 \mathrm{~Hz}$ & $1133 \mathrm{~Hz}$ \\
\hline Error & $1.9 \%$ & $6.7 \%$ & $2.2 \%$ & $0.9 \%$ \\
\hline Rotor\#4 & $1^{s t}$ & $2^{n d}$ & $3^{r d}$ & $4^{t h}$ \\
\hline Measured & $435 \mathrm{~Hz}$ & $735 \mathrm{~Hz}$ & $1228 \mathrm{~Hz}$ & $1802 \mathrm{~Hz}$ \\
\hline Predicted & $421 \mathrm{~Hz}$ & $798 \mathrm{~Hz}$ & $1190 \mathrm{~Hz}$ & $1783 \mathrm{~Hz}$ \\
\hline Error & $3.4 \%$ & $8.5 \%$ & $3.1 \%$ & $1.0 \%$ \\
\hline Rotor $\# 5$ & $1^{s t}$ & $2^{n d}$ & $3^{r d}$ & $4^{t h}$ \\
\hline Measured & $342 \mathrm{~Hz}$ & $676 \mathrm{~Hz}$ & $1121 \mathrm{~Hz}$ & $1624 \mathrm{~Hz}$ \\
\hline Predicted & $343 H z$ & $666 H z$ & $1093 \mathrm{~Hz}$ & $1655 \mathrm{~Hz}$ \\
\hline Error & $0.2 \%$ & $1.5 \%$ & $1.6 \%$ & $1.9 \%$ \\
\hline
\end{tabular}

mode shapes are shown in Figs. 6-10. The relative errors between the predicted and measured frequencies vary from $0.2 \%$ to $10 \%$ with a mean value equal to $3.1 \%$. Figs. $6-10$ show a good correlation between the predicted and measured mode shapes. Therefore, the identification method presented is more accurate than classical identification methods when considering restrictive assumptions on the mechanical behavior of a lamination stack mode of isotropic material. It shows that the FE model presented here is an efficient means of prediction the dynamics of this kind of real assembled structure.

\section{CONCLUSION}

The proposed optimization procedure was tested on several industrial induction motors with complex designs. An FE model 

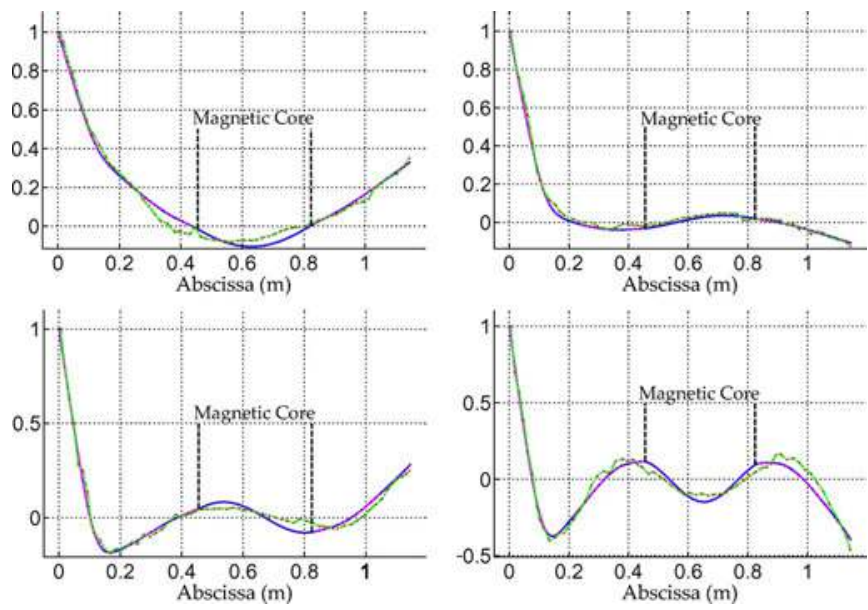

Fig. 6. First four (solid line) predicted and (dashed line) measured lateral mode shapes of the Rotor\#1.
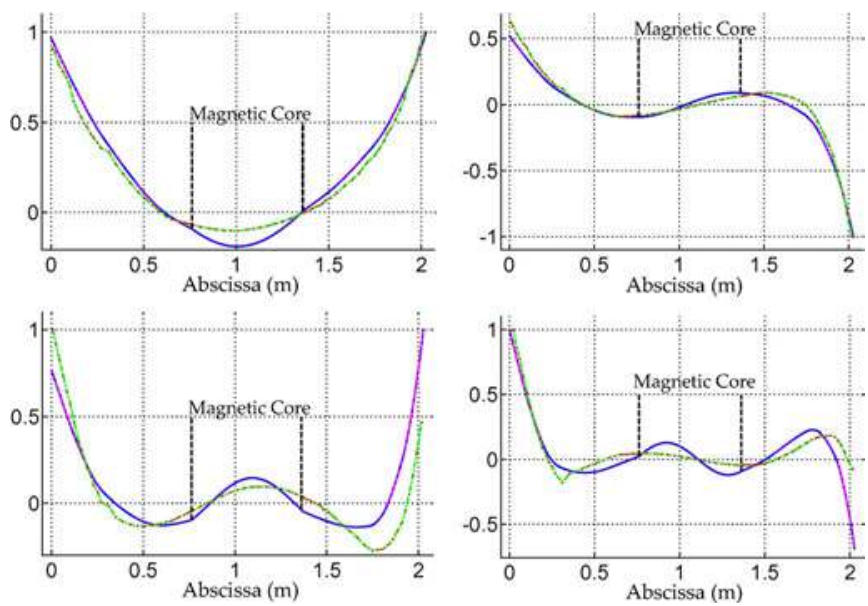

Fig. 7. First four (solid line) predicted and (dashed line) measured lateral mode shapes of the Rotor\#2.
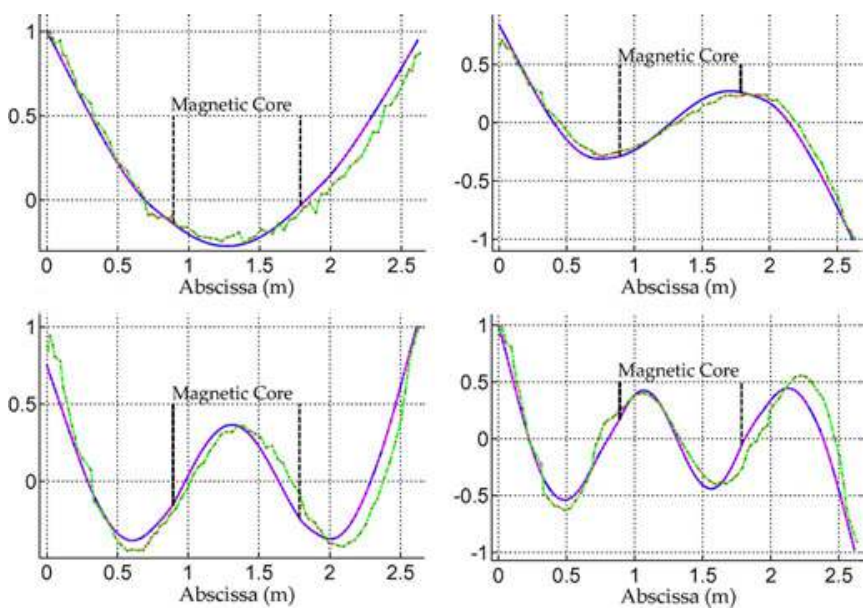

Fig. 8. First four (solid line) predicted and (dashed line) measured lateral mode shapes of the Rotor\#3.

was presented taking into account that MGV are assemblies made of different components including in particular a magnetic core. The prestressed tie rods were also modeled. It was
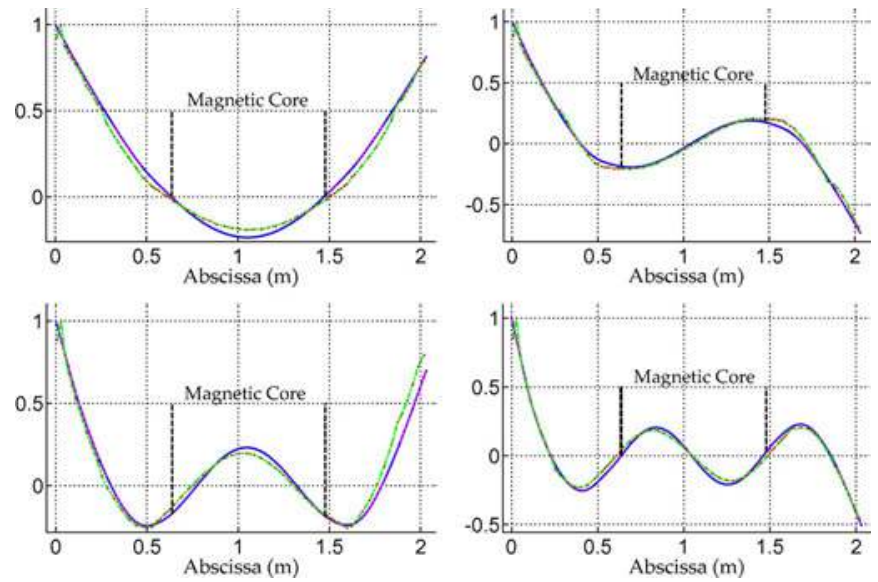

Fig. 9. First four (solid line) predicted and (dashed line) measured lateral mode shapes of the Rotor\#4.
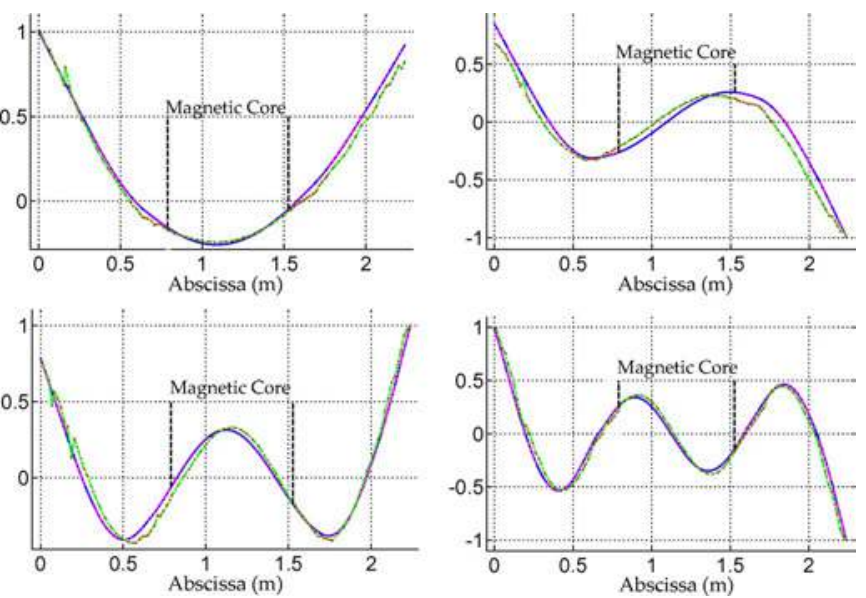

Fig. 10. First four (solid line) predicted and (dashed line) measured lateral mode shapes of the Rotor\#5.

shown that the identified equivalent constitutive properties of the lamination stack, i.e., its stiffness, depend on the rotor sizes. This procedure is very useful for formulating an FE model mainly based on beam elements containing few DOF, which is a great advantage for rotordynamics prediction, i.e., unbalanced and transient responses. The reliability of such a procedure will allow predicting the dynamic behavior of an MGV under development, operating at $6000 \mathrm{r} / \mathrm{min}$ in the $30-\mathrm{MW}$ range. This will permit the manufacturer to increase the reliability of this range of products.

\section{ACKNOWLEDGMENT}

The authors would like to thank Converteam for its support and permission to publish this paper.

\section{REFERENCES}

[1] R. Belmans, W. Heylen, A. Vandenput, and W. Geysen, "Influence of rotor-bar stiffness on the critical speed of an induction motor with an aluminium squirrel cage," Proc. Inst. Elect. Eng., vol. 131, pt. B, no. 5, pp. 203-208, Sep. 1984.

[2] S. Chang and D. Lee, "Robust design of a composite air spindle," Polym. Compos., vol. 23, no. 3, pp. 361-371, 2002. 
[3] J. McClurg, "Advantages of stiff shaft design on high speed, high horsepower squirrel cage induction motors and generators," in Proc. 34th APCI Conf., 1987, pp. 259-263.

[4] J. Ede, D. Howe, and Z. Zhu, "Rotor resonances of high-speed permanentmagnet brushless machines," IEEE Trans. Ind. Appl., vol. 38, no. 6, pp. 1542-1548, Nov./Dec. 2002.

[5] S. Garvey, J. Penny, M. Friswell, and A. Lees, "The stiffening effect of laminated rotor cores on flexible-rotor electrical machines," in Proc. 8th Int. Conf. Vibrations Rotating Machinery, 2004, pp. 193-202.

[6] Y. Chen, Y. Cheng, J. Liao, and C. Chiou, "Development of a finite element solution module for the analysis of the dynamic behavior and balancing effects of an induction motor system," Finite Elem. Anal. Des., vol. 44, no. 8, pp. 483-492, May 2008.

[7] Y.-C. Kim and K.-W. Kim, "Influence of lamination pressure upon the stiffness of laminated rotor," JSME Int. J., Ser. C, vol. 49, no. 2, pp. 426431, 2006.

[8] C. Lee and T. Kam, "Identification of mechanical properties of elastically restrained laminated composite plates using vibration data," J. Sound Vib., vol. 295 , no. 3-5, pp. 999-1016, 2006.

[9] J. Cugnoni, "Identification par recalage modal et fréquentiel des propriétés constitutives de coques en matériaux composites," Ph.D. thesis, Ecole Polytechnique Fédérale de Lausanne, Lausanne, Switzerland, 2005.

[10] X. Feng, J. Zhou, and Y. Fan, "Identifying sub-regional material parameters of concrete dams using modal data," Acta Mech. Solida Sinica, vol. 16, no. 1, pp. 88-94, 2003.

[11] I. Ojalvo, "Efficient computation of mode-shape derivatives for large dynamic systems," AIAA J., vol. 25, no. 10, pp. 1386-1390, 1987.

[12] R. Nelson, "Simplified calculation of eigenvector derivatives," AIAA J., vol. 14, no. 9, pp. 1201-1205, 1976.

[13] Y. Min, L. Zhong-Sheng, and W. Da-Jun, "Comparison of several approximate modal methods for computing mode shape derivatives," Comput. Struct., vol. 62, no. 2, pp. 381-393, 1997.

[14] J.-S. Przemieniecki, Theory of Matrix Structural Analysis. New York: Dover, 1985.

[15] G. Mogenier, R. Dufour, G. Ferraris Besso, L. Durantay, and N. Barras, "Optimization procedure for identifying constitutive properties of high speed induction motor," in Proc. ICEM, Vilamoura, Portugal, 2008, pp. 1-6.

[16] H. Nielsen, "Damping parameter in Marquardt's method," Tech. Univ. Denmark, Lyngby, Denmark, Tech. Rep. IMM-REP-1999-05, 1999.

[17] S. Villwock and M. Pacas, "Application of the Welch-method for the identification of two- and three-mass-systems," IEEE Trans. Ind. Electron., vol. 55, no. 1, pp. 457-466, Jan. 2008.

[18] M. Lalanne and G. Ferraris, Rotordynamics Prediction in Engineering., 2nd ed. New York: Wiley, 1998

[19] G. Cowper, "The shear coefficient in Timoshenko's beam theory," J. Appl. Mech., vol. 33, no. 2, pp. 335-340, 1966.

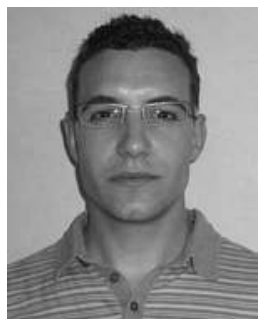

Guillaume Mogenier was born in Bourg en Bresse, France, in 1981. He received the M.Sc. degree in mechanical engineering from the University of Lyon 1, Lyon, France, in 2005. He is currently working toward the Ph.D. degree in rotordynamics of highspeed induction motors at the Institut National des Sciences Appliquées de Lyon (INSA Lyon), Villeurbanne, France.

He is currently with the Laboratoire de Mécanique des Contacts et des Structures (Unite Mixte de tifique 5259), INSA Lyon.

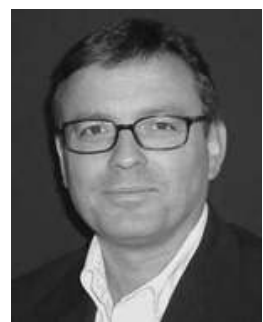

Régis Dufour received the Ph.D. degree from the Institut National des Sciences Appliquées de Lyon (INSA Lyon), Villeurbanne, France, in 1984.

$\mathrm{He}$ is a Professor of mechanical engineering at the University of Lyon, Centre National de la Recherche Scientifique (CNRS), INSA Lyon. He is the Deputy Director of the Laboratoire de Mécanique des Contacts et des Structures, Unite Mixte de Recherche, Centre National de la Recherche Scientifique 5259, comprised of 160 members, in which he manages the research group Dynamics and Control of Structures (40 members). His expertise concerns dynamic analysis of mechanical systems and structures: theory and experimentation. Nonlinear dynamics-rotordynamics-vibration isolation are his specific interests.

Dr. Dufour is an Editor-in-Chief of Mécanique \& Industries (EDP Sciences), Associate Editor of Shock and Vibration (IOS Press), and a member of the Editorial Board of the Journal of Vibration and Control (Sage Publications). He belongs to the Technical Committee of the International Federation for the Promotion of Mechanism and Machine Science Rotordynamics.

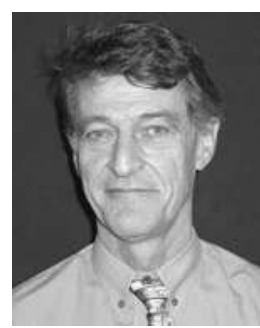

machinery dynamics.
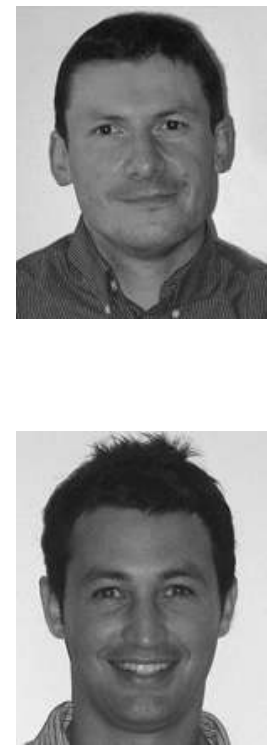

Guy Ferraris-Besso was born in Annecy, France, in 1943. He received the Ph.D. degree (3rd cycle) from the University of Lyon, Lyon, France, in 1982.

$\mathrm{He}$ joined the Institut National des Sciences Appliquées de Lyon (INSA Lyon), Villeurbanne, France, in 1973. In 1983, he became a Research Engineer with the Centre National de la Recherche Scientifique, and was detached to INSA Lyon. Since 1994, he has been a Professor at INSA Lyon. His research interests are mechanics-computer science-engineering vibrations, and rotating

Lionel Durantay was born in Nancy, France, in 1965. He received the M.Sc. degree in mechanical engineering from the Ecole Nationale Supérieure d'Electricité et de Mécanique, Nancy, in 1989, and the $\mathrm{Ph}$.D. degree in mechanics from the Institut $\mathrm{Na}$ tional Polytechnique de Lorraine, Nancy, in 1994.

$\mathrm{He}$ is currently the R\&D Director of the Rotating Machines activities for Converteam Southern Europe Middle East, and Africa, Champigneulles, France.

Nicolas Barras was born in Versailles, France, in 1982. He received the M.Sc. degree in mechanical engineering from the Institut National des Sciences Appliquées de Lyon, Villeurbanne, France, in 2005.

$\mathrm{He}$ is currently with Converteam SEMEA, Champigneulles, France, as Lead Engineer for highspeed induction motors. 